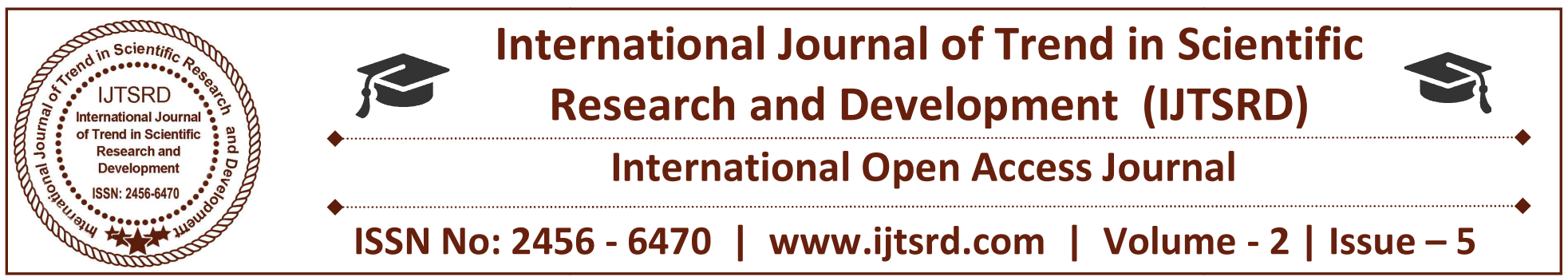

\title{
Review on Congestion Control Algorithm for VANET
}

\author{
Manoj Rathore ${ }^{1}$, Prof. Anuj Kumar Pal ${ }^{2}$ \\ ${ }^{1}$ Research Scholar, ${ }^{2}$ Assistant Professor \\ Department of Computer Science and Engineering, \\ Bansal Institute of Research and Technology, Bhopal, India
}

\section{ABSTRACT}

Vehicular ad hoc networks (VANETs), which is a subclass of Mobile ad hoc networks, have recently been developed as a standard means $c$ of communication among moving vehicles because it has tremendous potential to improve vehicle and road safety, traffic efficiency. VANET applications have the characteristics such as fast change of topology, bandwidth limitation and lack of central coordination that causes network congestion which restricts the network performance. Number of solutions proposed to overcome these challenges and to reduce congestion on VANET. These solutions include congestion control algorithms, some of them based on maintaining the beacon load below certain threshold value by adjusting transmit power or transmit rate or both. And others are based on utility function, carrier sense threshold, prioritization or a combination of them. Hence, this study takes a closer look at existing congestion control algorithms to solve congestion problems because it affects the performance of safety messages. The study further exposes the weaknesses and advantages of some of these congestion control algorithms, which can be helpful to tackle with the problems of congestions in VANETs.

KEYWORD: VANET, Beacon messages, Event driven messages, Congestion control, IEEE 802.11p

\section{INTRODUCTION}

The networks with the absence of any centralized or pre-established infrastructure like access points in managed wireless networks or routers in wired networks called Ad hoc networks [8]. Such wireless ad hoc networks can be categorized via their application, such as Wireless Mesh Networks (WMN), Mobile Ad Hoc Networks (MANET)
Wireless Sensor Networks (WSN), and Vehicular Ad hoc Networks (VANETs). VANET is a particular type of MANET, that vehicles play the role of nodes in it. As opposed to MANET, vehicles move on predefined roads and velocity depends on the speed signs. Considering providing comfort and safety to the road users, VANET regarded as one of the influencing areas in advancement of the intelligent Transportation System (ITS).

The basic target of VANET is to increase safety of the road users and comfort of the passengers. There are many challenges in VANET that have to be resolved to offer reliable services such as routing, security, and quality of service. Due to many issues such as Inaccurate State of Information, dynamically Varying Network Topology, Absence of Central Coordination, Hidden Terminal Problem, Limited Resource Availability Error Prone Shared Radio Channel, and Insecure Medium, therefore, supporting Quality of Service (QoS) is a challenging task. Many approaches proposed to improve the QoS in VANETs. For providing better QoS, congestion control algorithms are widely used. There are two types of messages to enable safety applications. On the one hand, cooperative awareness messages (CAMs), also known as beacons, broadcasted periodically by all nodes on the control channel, in order to receive and provide status information on presence, geographical position and movement of neighboring nodes, and service announcements to/from those nodes. On the other hand, emergency event-driven messages transmitted when it notice abnormal or hazardous condition, in order to inform surrounding nodes about it. [7] The challenge regarding this beaconing activity will be to control the load in the channel in order to stay away 
from channel congestion. Congestion control achieve by making efficient use of available channel bandwidth

VANET belongs to wireless communication networks area. The Federal Communication Commission (FCC) allocates the frequency spectrum for VANET's wireless communication. Then the Commission in 2003 established the Dedicated Short Range Communications (DSRC) Service. The DSRC is a communication service, which utilizes for public and private safety and uses the frequency range of 5.850$5.925 \mathrm{GHz}$ [1]. The DSRC designed in multi-channel system. The FCC divided the DSRC spectrum into seven channels so that each of them has $10 \mathrm{MHz}$ bandwidth. Six of them identified as Service Channels (SCH), and one of them then identified as the Control Channel $(\mathrm{CCH})$, as shown in Fig. 1. The CCH channel is used for safety messages, while SCH channels are used for non-safety as well as WAVE-mode messages or services[3].

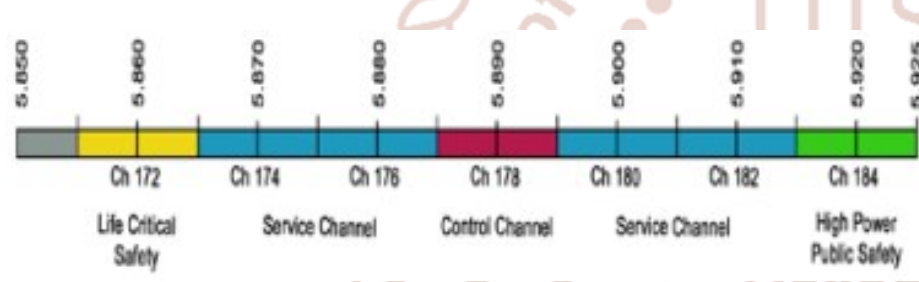

Figure 1: DSRC channels

In high traffic, a large number of vehicles broadcast beacon messages at a high frequency, which can easily congest the $\mathrm{CCH}$ channel. The periodic messages are broadcast may lead to broadcast storm flooding problem in VANETs. It is very important to keep the $\mathrm{CCH}$ channel free from congestion in order to ensure timely and reliable delivery of event-driven safety messages In order to avoid congestion of $\mathrm{CCH}$ channel and delay of event-driven safety message, a reliable and efficient congestion control algorithm is needed.[4]

This paper gives the detailed study of various congestion control schemes and reveals the advantages and disadvantages of them. It does thorough analysis of the parameters, which affect congestion.

\section{PARAMETERS AFFECTING CONGESTION}

Based on number of researches, various parameters, which affect congestion, analyzed and the tread off between them derived. The parameters that directly affect the channel congestion are termed as primary parameters such as transmit power, transmit rate and beacon frequency; whereas the derived parameters depend on the primary parameters, which are fairness, prioritization, and utility function.

\subsection{Transmit Power}

Transmit power is defined as power content of each node. With transmit power control; beacon reception rate decreases hence event driven reception increases. This further reduces channel busy time and results in the congestion reduction.[1]

\subsection{Transmit Rate}

It is defined as the rate at which packets are transmitted over the channel. With transmit rate control, event driven probability is increased this reduces $\mathrm{CBT}$ and reduces channel congestion. [1]

\subsection{Beacon Frequency}

Beacon frequency is the number of beacon messages transmitted by a node per unit time. In dense network beacon frequency reduces; as density decreases, the beacon frequency increases. This frequency control results in improvement of beacon reception probability this reduces channel load and hence channel congestion. [5]

\subsection{Fairness}

Fairness deals with providing fair portion of available resources to each node so that it can get equal opportunity over channel. Fairness maximizes the minimum transmit power, it increases individual coverage and hence road safety.

\subsection{Prioritization}

Prioritization means assigning more priority to event driven messages than beacon message, this increases safety and reduces congestion.

\subsection{Utility Function}

The utility function used to determine utility of transmitting data packet of a particular application at current point in time. The application specific utility function calculated for each packet and encoded in packet header for estimating utility of individual data packet at each node .based on estimated utility of data packets. Each node adapts its own data rate. In case of congestion, it drops lower utility packets.

\subsection{Node Density}

VANET is affected by characteristics of traffic flow. Vehicle (node) density influences average speed of 
node and thus affecting network mobility. In general, when node density increases; it increases the packet transmission rate, which ultimately increases channel load and results in congested channel. This parameter generally use for simulation and analysis purpose.

We analyzed these parameters thoroughly in following section with the help on different congestion control algorithms. These algorithms along with one can use different congestion detection methods or more parameters mentioned above.

\section{DISCUSSION AND OPEN POINT}

As we can see all the presented techniques are aimed to face vehicle traffic congestion and monitoring traffic conditions. All proposed techniques are based on $\mathrm{V} 2 \mathrm{~V}$ communication that performs Infrastructureless approach of Traffic Information System. These techniques are COC, SOTIS, Traffic View, Miller, StreetSmart, Vaquar and Lin. All mentioned techniques can be classified into two main categories according to type of message transmission: Periodic message transmission and Non periodic message transmission.

Table 1 summarizes the previous discussed techniques, gives some metrics for comparing their performance, and reviews the existing V2V-based traffic congestion techniques based on their detection capabilities and the type of traffic information they provide. Congestion detection indicates the capability of the technique to monitor traffic conditions and detect congestion situations. Detection correlation refers to whether individual congestion estimates are correlated or not among several vehicles to reach a consensus decision. Congestion level and Traffic jam length refer to the technique's capability to classify the detected traffic jam's congestion level (or intensity) and quantify its length. Limited overhead indicates whether the technique is capable to limit the generation of communications overhead to only situations of abnormal traffic conditions (e.g. traffic jam). Finally, Dissemination indicates whether the technique includes the capacity to select the vehicle that will be in charge of disseminating the detected traffic congestion conditions to approaching vehicles or road authorities (e.g. through nearby road side units or cellular links).

This capacity would avoid the possibility that multiple vehicles detecting the same traffic congestion conditions generate redundant messages that could overload the communications channel.

1) The technique provides traffic information such as the vehicles' speed and/or traffic density. However, it does not classify the congestion level based on the collected traffic measurements.

2) The technique has been designed to monitor road traffic conditions, but is currently not able to explicitly detect congestion situations.

3) The detection correlation is based on a voting process carried out among vehicles located in the onehop neighborhood; no multi-hop correlation is applied.

4) The technique does not explicitly provide the traffic jam length. Such length could be indirectly inferred from the locations of the jam header and tail, but this would require an evolution of the original proposal.

There other metrics must be taken in account when we discussing any techniques, those metrics effect on the design and performance of each technique or system. Such metrics are Ease of deployment and cost effectiveness, Reliability, Privacy and Security.

\subsection{Ease of Deployment and Cost Effectiveness}

As we discuss technique based onV2V communication so they don't use any infrastructure such as roadside units or any centralized points so it much cheaper for deployment than infrastructure based techniques. For techniques such as COC, TrafficView and SOTIS they depend completely on $\mathrm{V} 2 \mathrm{~V}$ communication so it's inexpensive for deploying. Also (Vaqar and Basir) technique and (Lin and Osafune) technique. On other hand Miller and StreetSmart they use some kind of servers for getting more accuracy in measurements they cost more and more complicated to be deployed in real world. 
Table 1: Traffic Monitoring Techniques

\begin{tabular}{|c|c|c|c|c|c|c|}
\hline & & & & \\
\hline COS & Yes & Yes & Yes & Yes & No & No \\
\hline Traffic View & Yes & Yes & Yes & Yes & No & No \\
\hline SOTIS & Yes & Yes & Yes & Yes & No & No \\
\hline Miller & Yes & Yes & Yes & Yes & No & No \\
\hline Street & Yes & Yes & Yes & Yes & Yes & No \\
\hline Smart & Yes & No & Yes & No & Yes & No \\
\hline Vaquar \&Basir & Yes & Yos \\
\hline Lin \& Osafune & Yes & Yes & No & No & Yes & Yes \\
\hline
\end{tabular}

\subsection{Reliability}

Reliability is the ability of the system component to function under stated conditions. For our techniques where they based on distributed approach (Infrastructureless) approach so if one or more node (vehicle) failed will not affect on the whole systems. For Miller and StreetSmart techniques, they are not reliable like other techniques such as COC, TrafficVIEW, SOTIS where in their architecture depend on some servers so if one server failed the whole system will be affected. Although Miller technique uses both V2V and V2I communication together when a failure occurs the systems use only V2V communication.

\subsection{Privacy and Security}

When new cooperative networks systems such as VANETs are deployed, a major concern for users is the loss of privacy, after all nobody wants his/her vehicle to be telling everybody where it is at all times. Most academic work on congestion detection in VANETs ignore this important fact and use unique vehicle IDs that persist over time making it possible for a malicious node to track the location of a vehicle, for this reason, the solution proposed in this work does not require vehicles to maintain unique IDs. Along with privacy, security is a legitimate concern for real-world systems. Preventing users from abusing or sabotaging the system is paramount. Several initiatives have been developed in recent years to provide secure V2V communications [5] [6] [7] [8]. Techniques such as SOTIS, StreetSmart and Miller have no IDs for vehicles but techniques such as COC,
TrafficView, Vaqar and Basir and Lin and Osafune each vehicle has its own ID.

\section{CONCLUSION}

Vehicular traffic congestion is a major problem associated with vehicular traffic, which has been attracting the extensive attention of research in the field of VANETs. Vehicular congestion estimation shall not only provide a basis for development of traffic monitoring application but also aid in development of other applications which can exploit traffic congestion information for other goals in VANETs such as disseminating information, suggesting alternate driving route etc. So this survey discusses different approaches of traffic information systems and how the Infrastructureless approach is the more one to be used in transportation system. Then it discusses different techniques and systems developed by many researchers for traffic congestion and monitoring systems. So for building traffic system to detect congestion and allow drivers to avoid it, the most powerful option is Infrastructureless system that based only on $\mathrm{V} 2 \mathrm{~V}$ communication without any infrastructure. Those systems don't require large investment so can be easily deployed.

\section{REFERENCES}

1) Bhargavi Goswami and Saleh Asadollahi, "Novel Approach to Improvise Congestion Control over Vehicular Ad-Hoc Networks (VANET)", IEEE Conf on ITS, 2016.

2) Wellington Simbarashe Manjoro, Mradul Dhakar and Brijesh Kumar Chaurasia, "Traffic Congestion Detection using Data Mining in VANET", IEEE Students' Conference on Electrical, Electronics and Computer Science, 2016.

3) Cynthia Jayapal and Sujith Roy, "Road Traffic Congestion Management Using VANET, International Conference on Advances in Human Machine Interaction (HMI - 2016).

4) Mattia Minelli and Samir Tohm "The Potential of Transmit Data Rate Control for Channel Congestion Mitigation in VANET", IEEE Trans on Vehicular Tech. 2016.

5) Arrate Alonso and Christoph F. Mecklenbrauker "Dependability of Decentralized Congestion Control for varying VANET density", IEEE Transactions on Vehicular Technology, 2016. 
6) Abdellah IDRISSI, Abdelquoddouss LAGHRISSI, Sara RETAL and Hajar REHIOUI "VANET Congestion Control Approach using Empathy", IEEE Conf on ITS, 2015.

7) Heba El-Sersy and Ayman El-Sayed, "Survey of Traffic Congestion Detection using VANET", Communications on Applied Electronics (CAE), 2015.

8) Miao Wang, Hangguan Shan, Rongxing Lu, Ran Zhang, Xuemin (Sherman) Shen and Fan Bai "Real-Time Path Planning Based on HybridVANET-Enhanced Transportation System", IEEE Transactions on Vehicular Technology, 2015.
9) Shruti R. Kolte and Mangala S. Madankar "Adaptive Congestion Control for Transmission of Safety Messages in VANET", International Conference for Convergence of Technology, 2014.

10) Shruti R. Kolte and Mangala S. Madankar, "A Design Approach of Congestion Control for Safety Critical Message Transmission in VANET", Fourth International Conference on Communication Systems and Network Technologies, 2014.

11) Atanu Mondal and Sulata Mitra, "Dynamic and Distributed Channel Congestion Control Strategy in VANET", IEEE Conf on Embedded System, 2014. 